\title{
EXTENDED SUPRASTERNAL PUNCTURE
}

BY

\author{
J. P. BLACKBURN AND P. R. FLEMING
}

From the Department of Clinical Measurement, Westminster Hospital, and the Professorial Medical Unit, Westminster Medical School, London S.W. 1 .

Received January 9, 1964

The technique of suprasternal puncture of the left atrium and great vessels was first described by Radner nearly ten years ago in a series of publications in which he outlined the successive stages in its development (Radner, 1953, 1954a, b, and c, 1955). He had been using the suprasternal approach for aortic puncture and had found that the needle would occasionally pass behind the aorta and enter the left atrium. During the further development of left atrial puncture he found that, in 3 of his first 6 cases, the needle passed through the pulmonary artery before entering the left atrium. Impressed by the simplicity and safety of these procedures, he subsequently described the technique of "extended suprasternal puncture" in which the aorta, pulmonary artery, and left atrium are punctured successively.

In this paper we propose to describe our experience for the past four years with suprasternal puncture, either alone or in combination with other methods of left heart catheterization. We shall also describe the instrumentation that has been developed to allow the maximum information to be obtained from these investigations.

\section{SuBjeCtS AND Methods}

Suprasternal puncture has been carried out in 52 patients whose ages ranged from 8 to 65 . In 50 there was evidence of mitral valve disease either alone or in association with aortic valve disease: in these patients the purpose of the procedure was to determine the relative significance of the valvular lesions. In addition, one patient with cor pulmonale was investigated to determine the pulmonary arterial pressure and, in a case of pure aortic stenosis, suprasternal aortic puncture was carried out together with percutaneous left ventricular puncture.

Apparatus. The needle* used is short bevel, $18 \mathrm{~cm}$. long, $0.8 \mathrm{~mm}$. in outside diameter and $0.4 \mathrm{~mm}$. inside diameter (Fig. 1). This needle is connected via a two-way tap to a $2 \mathrm{ml}$. syringe; this is normally filled with saline for intermittent flushing of the needle but can also be used for injection of indicators or for taking blood samples. The other outlet from the tap is connected via four feet of polytetrafluoroethylene tubing, of inside diameter about $2.5 \mathrm{~mm}$., to a Statham P23G strain gauge pressure transducer. When carefully filled, this system is capable of a frequency response in excess of $30 \mathrm{c} / \mathrm{s}$.

Signals from the pressure transducers and from electrocardiograph electrodes and phonocardiograph microphones pass to sockets on the x-ray table and thence via underfloor cables to wall sockets above the instrumentation bench; thus trailing cables are eliminated. The signals then pass via the appropriate preamplifiers to a junction box (Fig. 2). This was designed to overcome the difficulties not infrequently encountered when operating different items of commercial equipment in combination. The junction box will accept up to 10 inputs ( 1 for an electrocardiogram, 3 for phonocardiograms, 4 for pressure signals, and

* Made by Genito-Urinary Mfg. Co. 


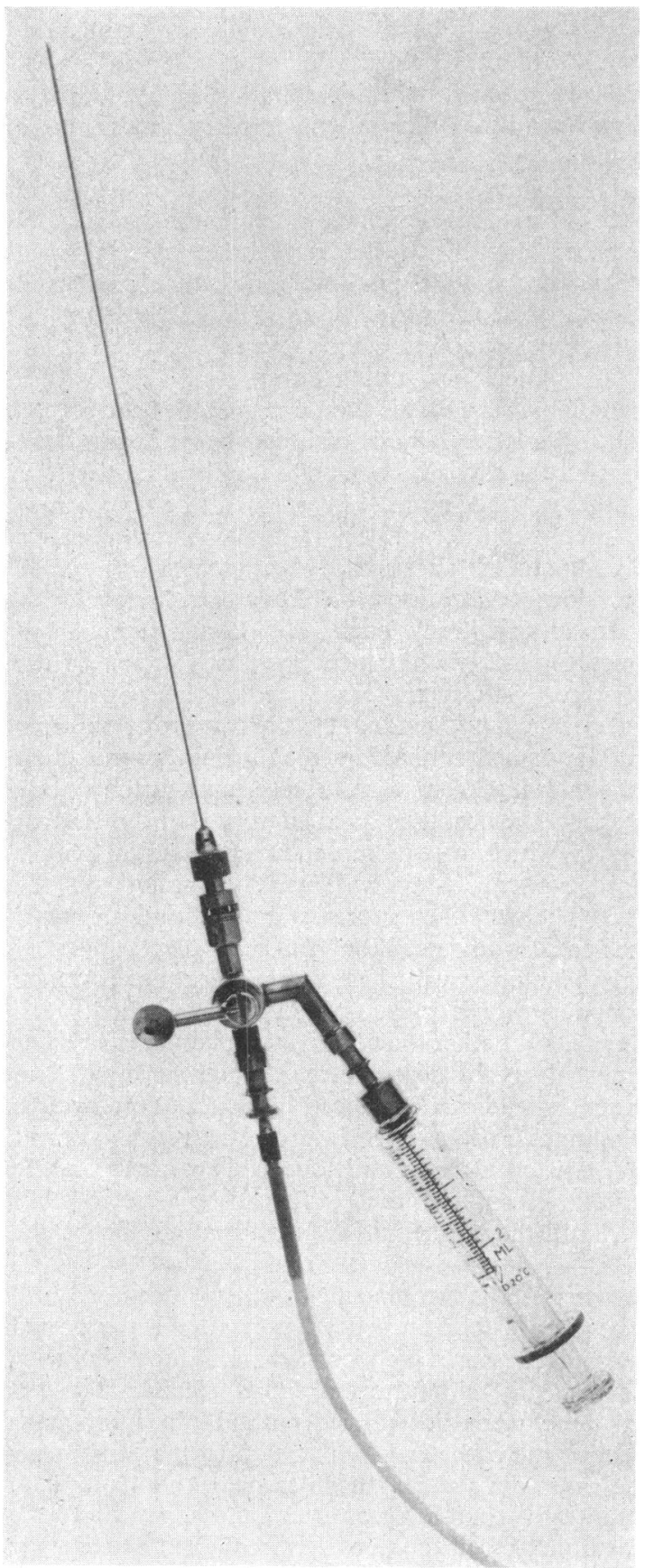

FIG. 1.-Suprasternal puncture needle attached via two-way tap to syringe and polytetrafluoroethylene tubing.

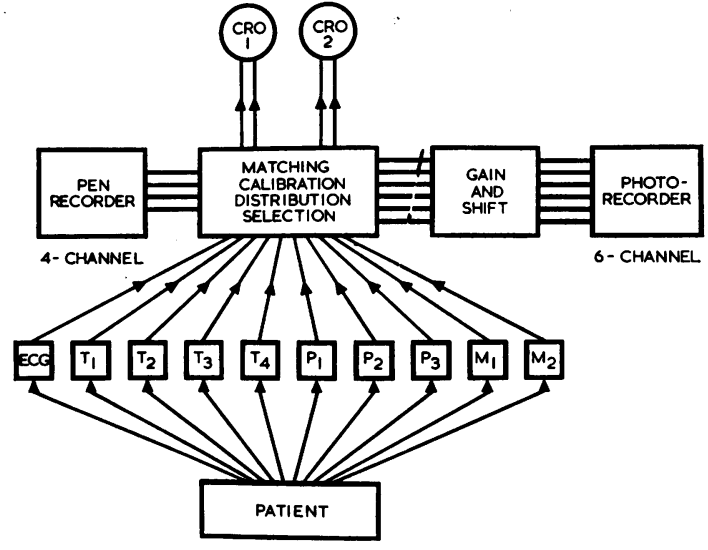

FIG. 2.-Block diagram of instrumentation. Underfloor cables link patient and pre-amplifiers. T1-4-pre-amplifiers for pressure transducers. P1-3-phonocardiograph pre-amplifiers. M1-2pre-amplifiers for miscellaneous inputs.

2 for miscellaneous inputs) and switch them in any desired combination to the recorders. These consist of a four-channel Sanborn pen recorder; a sixchannel N.E.P. photographic recorder* equipped with 1 galvanometer for the electrocardiogram, 2 for phonocardiograms, and 3 for pressure recordings or other use; 2 double beam cathode ray oscilloscopes $\uparrow$ for continuous monitoring. All the pens and galvanometers can be adjusted independently for position and gain and any of the inputs can be displayed instantly on the monitor screens. High input impedance cathode followers are used to separate the stages so that switching different combinations of recorders and oscilloscopes does not affect the calibration of those already in use. Built-in attenuators are used to equalize the outputs of the different pre-amplifiers; thus changes in gain are not necessary when switching different signals on to the recorders.

Procedure. Preliminary postero-anterior and lateral chest films are taken and, on the latter, the positions of the aortic arch, the pulmonary artery, and the left atrium are noted; the angle at which the needle should be passed can then be estimated (Fig. 3). If the left atrium is not clearly delineated, its position can be inferred from the course of the left main bronchus which lies along its upper border. The bleeding, clotting, and prothrombin times and platelet count are also estimated and a significant abnormality in any of these is regarded as a

* Recorder Series 100. Honeywell Controls Ltd., Medical Division.

$\dagger$ Type 680. Honeywell Controls Ltd., Medical Division. 


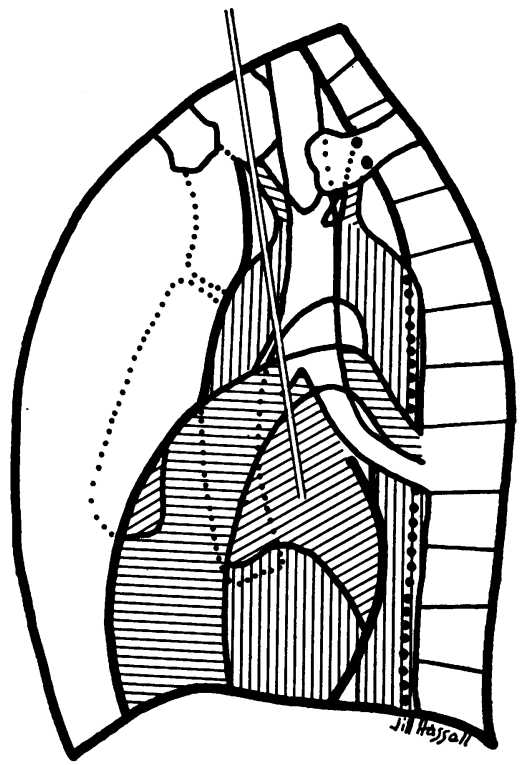
show course of needle through aorta and pulmonary artery to enter left atrium.
Fig. 3.-Diagram of lateral radiograph of chest to

contraindication to suprasternal puncture. The usual premedication is a small dose of morphine or papaveretum with a full dose of atropine or scopolamine given 45 minutes beforehand. Light general anæsthesia was used for the investigation of a child of 8 years; for all the other patients local anæsthesia alone was used.

In the investigation of patients with mitral and aortic valve disease, suprasternal puncture has usually been combined with other methods of left heart catheterization. Before discussing these combined procedures, however, a more detailed account of suprasternal puncture will be given.

The patient lies supine on an $\mathrm{x}$-ray table with a small pillow beneath his shoulders and his head turned well to the left. If he is troubled by orthopnœa the procedure is carried out with the table tilted to 15 or 20 degrees. The point of insertion of the needle is at the medial border of the right sternomastoid two finger-breadths above the sternoclavicular joint. After local infiltration with 2 per cent lignocaine, a broad cutting needle such as a Bonney's needle is passed for 1-2 cm. along the proposed course of the puncture. The suprasternal needle is held by the tap and syringe and, guided by the fingers of the free hand, is passed behind the manubrium sterni at an angle of about $30^{\circ}$ with the horizontal; the angle varies somewhat from case to case, and further study of the lateral chest radiograph may be necessary at this stage. The needle is directed very slightly towards the left, unless the patient has had a mitral valvotomy in which case a more directly sagittal course should be followed. After the needle has been advanced 5-6 cm. its tip will usually impinge on the aortic arch, the pulsations of which are easily perceptible. The wall of the aorta is pierced and an aortic pressure pulse recorded. The pressure is monitored as the needle is advanced for a further $2-3 \mathrm{~cm}$. obliquely across the aortic arch. After the needle has passed through the inferior wall of the aorta, pulsations continue to be felt, and it is not usually possible to recognize the pulmonary arterial pulse as that vessel is reached. It is, however, often entered as the needle is advanced more deeply. Whether the pulmonary artery is entered or not, a further advance to a total depth of $12-15 \mathrm{~cm}$. will almost always cause the needle to enter the left atrium.

Once the needle is cleanly within that chamber the patient's head can be placed in a more comfortable position; it can be left in the left atrium for as long as $\mathbf{3 0}$ minutes if necessary. If any of the vessels are not punctured the needle can be withdrawn to the level of the suprasternal notch and advanced again at a slightly different angle. Alternatively, if the left atrial pressure alone is required, the needle can be directed more posteriorly, parallel to the anterior border of the trachea, until the left atrium is punctured just below the level of the tracheal bifurcation.

The value of suprasternal puncture is considerably enhanced if it is combined with other procedures, and to illustrate this a brief outline of our present practice will be given. Right heart catheterization is performed and a needle placed in the brachial artery. Pulmonary vascular pressures are recorded and if the pulmonary "capillary" pressure pulse is of good quality and the pressure clearly within normal limits, significant mitral valve disease is presumed to be absent and suprasternal puncture is not carried out. If the pulmonary "capillary" pressure pulse is in any way abnormal, however, the catheter is withdrawn to the pulmonary artery and the investigation proceeds as follows. A Kifa radio-opaque polythene catheter (Ödman, 1959) is introduced by the Seldinger technique (Seldinger, 1953) into the right femoral artery and advanced to the ascending aorta. Suprasternal puncture is then performed and, when the needle is in the left atrium, the aortic catheter is passed into the left ventricle. The diastolic gradient across the mitral valve can now be recorded and blood samples withdrawn from the brachial and pulmonary arteries for cardiac output determination. If it is not possible to pass the aortic catheter into the left ventricle, percutaneous left ventricular puncture (Brock, Milstein, and Ross, 1956) is performed. The combination of retrograde aortic catheterization, suprasternal puncture, and left ventricular puncture is particularly valuable in cases of mitral and 
aortic valve disease as the pressure gradients across the mitral and aortic valves can be recorded simultaneously (Hansen et al., 1961). The investigation is usually completed by angiocardiography, the contrast medium being injected via the aortic catheter into the left ventricle or ascending aorta for the assessment of mitral and aortic incompetence.

Following the investigation a chest radiograph is taken and the patient's pulse rate and blood pressure taken every 30 minutes for 6-12 hours. A second radiograph is usually taken $24-48$ hours later.

\section{RESULTS .}

Out of the 52 patients investigated, the aorta was punctured in $38(73 \%)$, the pulmonary artery in $28(54 \%)$, and the left atrium in $49(94 \%)$. Puncture of the aorta, pulmonary artery, and left atrium successively was attempted in 39 patients and was completely successful in 22; in 14 the aorta and left atrium were punctured at the first attempt and no further attempts to reach the pulmonary artery were made as pulmonary arterial pressure had already been recorded via the catheter. In 2 patients both the aorta and the pulmonary artery were missed and the needle passed directly into the left atrium. In one patient only was the investigation unsuccessful; the pulmonary artery was punctured but, despite several attempts, neither the aorta nor the left atrium was entered. In 11 patients the needle was deliberately directed posteriorly to pass behind the great vessels and enter the left atrium only; this was usually on account of severe pulmonary hypertension. This manœuvre was successful in 7 patients, but in the other 4 the needle entered the pulmonary artery before puncturing the left atrium. Of the remaining 2 patients, one had cor pulmonale and no attempt was made to reach the left atrium after pulmonary arterial pressure had been recorded, and the other had pure aortic stenosis and the aorta only was punctured, simultaneously with left ventricular puncture.

The type of records obtained by these procedures is illustrated in Fig. 4-6. The superior quality of records obtained via the needle compared with those obtained via a catheter is apparent. The value of the combined procedures in obtaining simultaneous records of the pressures on either side of a stenosed valve is also illustrated; this is particularly valuable in patients with atrial fibrillation in whom the variations in cycle length prevent accurate superimposition of pressure pulses recorded consecutively.

Few complications were encountered in this series. In several patients a small amount of blood-stained pericardial fluid was found at operation; in one patient 50-100 ml. of more heavily blood-stained fluid was present without any previous untoward symptoms. One patient was troubled with nausea and retching before and during suprasternal puncture; this could conceivably have been due to the premedication. In two patients the systolic blood pressure fell $30-40 \mathrm{~mm}$. $\mathrm{Hg}$ for a few minutes but rose again without active treatment after withdrawal of the needle. Several patients had slight retrosternal discomfort during and after the procedure but in only two did this require analgesic drugs; in one of these there were no other complaints, but the other patient had some bradycardia without hypotension and subsequent chest radiograph showed a small pneumomediastinum; it seems probable that the needle entered the left bronchus at some stage. By the next day the patient had no symptoms and the air was rapidly absorbed. One patient, who

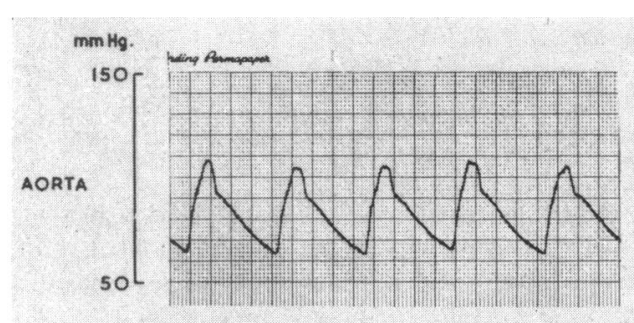
3L

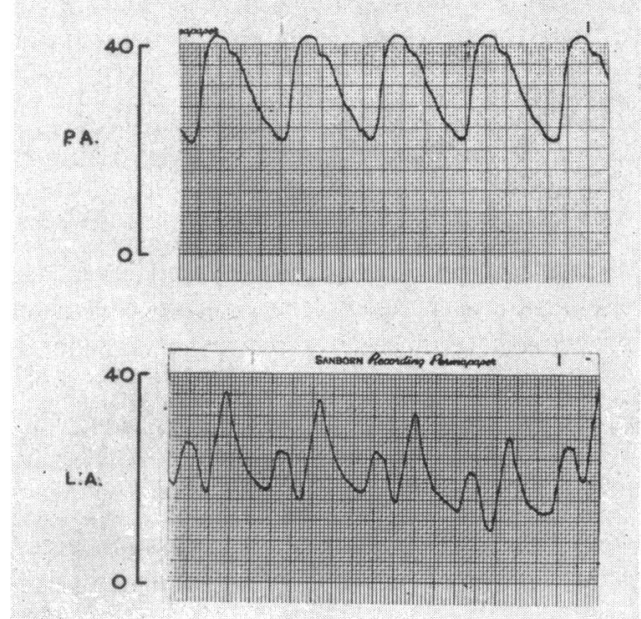

Fig. 4.-Successive puncture of aorta, pulmonary artery, and left atrium in a case of severe mitral stenosis. 


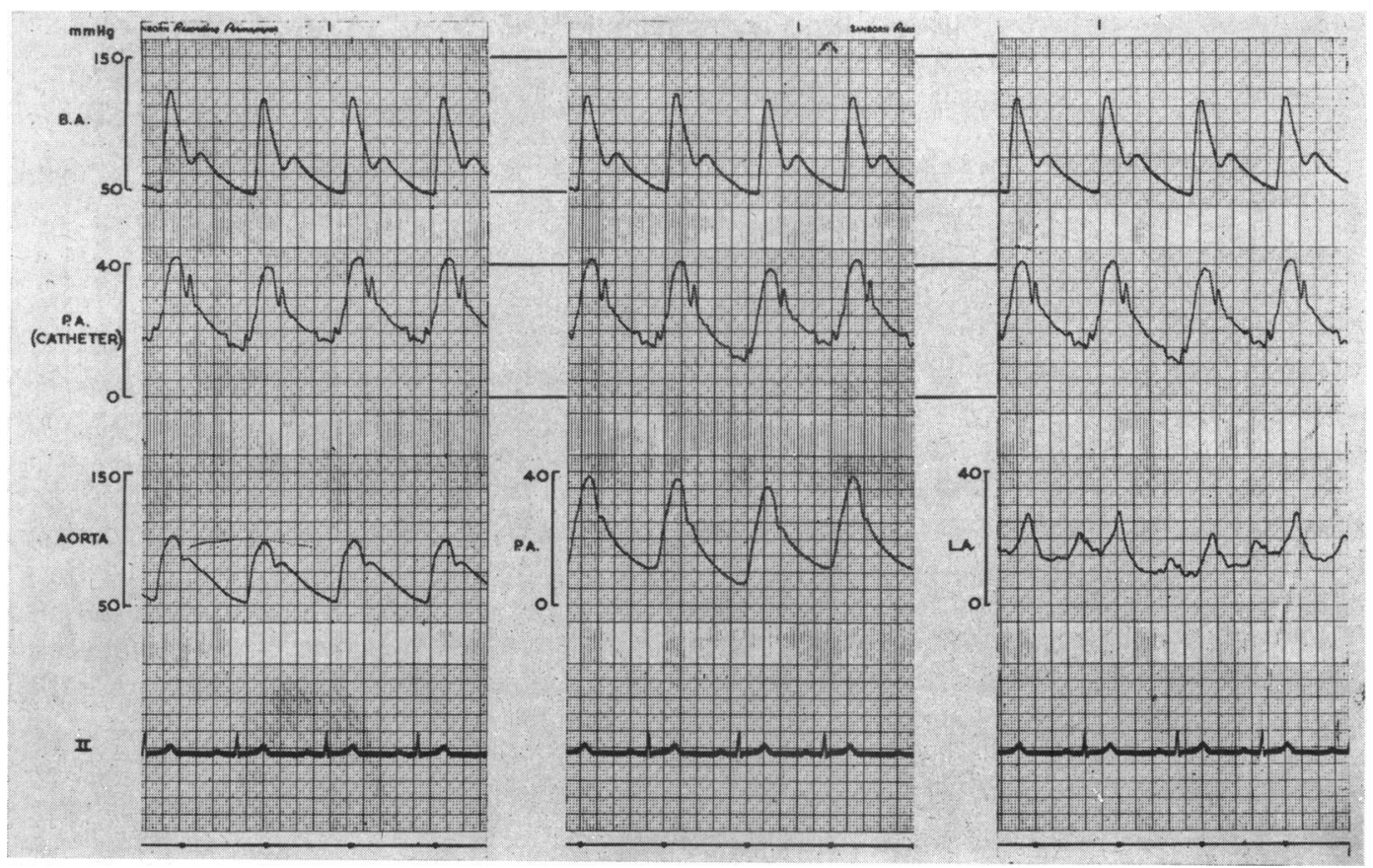

FIG. 5.-Brachial arterial puncture, pulmonary arterial catheterization, and suprasternal puncture of aorta, pulmonary artery, and left atrium in a case of moderately severe mitral stenosis. The artefacts due to catheter movement in the pulmonary arterial pressure pulse are not seen in the record obtained via the needle.

was investigated early in the series, had a fairly prolonged period of what can only be described as "medullary depression". About four hours after the end of the procedure, bradycardia, systemic hypotension, and bradypnca were noted. These were very slowly progressive over the next 6 hours. The patient slept for part of this period and Cheyne-Stokes respiration was present at this time. She was easily roused, however, and after two doses of nikethamide, $2 \mathrm{ml}$. intramuscularly, she slowly returned to normal. No definitive explanation for this episode was found but it was thought possible that it might have been due to a small hæmorrhage into the aortic adventitia, causing stimulation of baroceptor nerve endings. In the experimental animal, stimulation of the central end of the cut aortic "depressor" nerve causes reflex bradycardia, hypotension, and depression of respiration (Keele and Neil, 1961).

The maintenance of a stable state during hæmodynamic studies is of great importance, and an attempt has been made to assess the disturbing effect of suprasternal puncture. In Fig. 7 the heart rate and systemic arterial pressure (brachial arterial or aortic) before and during suprasternal puncture and the arteriovenous oxygen difference before and immediately after are plotted. In several cases other procedures intervened between right heart catheterization and suprasternal puncture, and these have been omitted.

\section{Discussion}

Although it is now ten years since suprasternal puncture was first described it has received scant attention; in some of the few reports adverse comments have been made. It is, therefore, appropriate to attempt to assess the value of this technique compared with other, more widely used, methods of left atrial puncture.

The ideal technique for this purpose should be completely safe, technically simple with a high success rate even in inexperienced hands, and it should produce no disturbance in the stable state. 


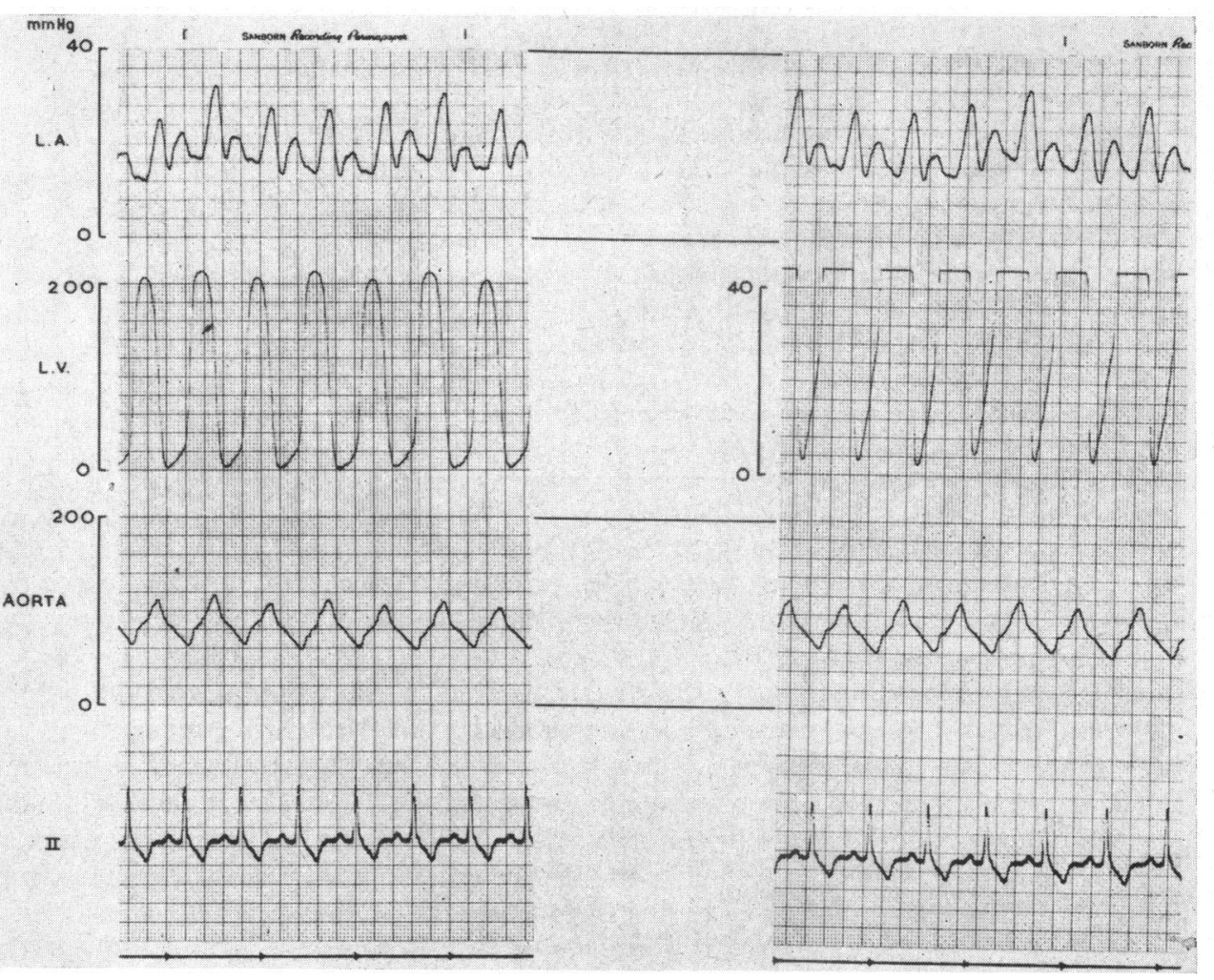

FIG. 6.- Retrograde aortic catheterization from femoral artery, suprasternal puncture of left atrium, and percutaneous left ventricular puncture in a case of severe aortic stenosis and mild mitral
stenosis. In the right-hand records only the diastolic portion of the left ventricular pressure pulse has been recorded for comparison with the left atrial pressure.
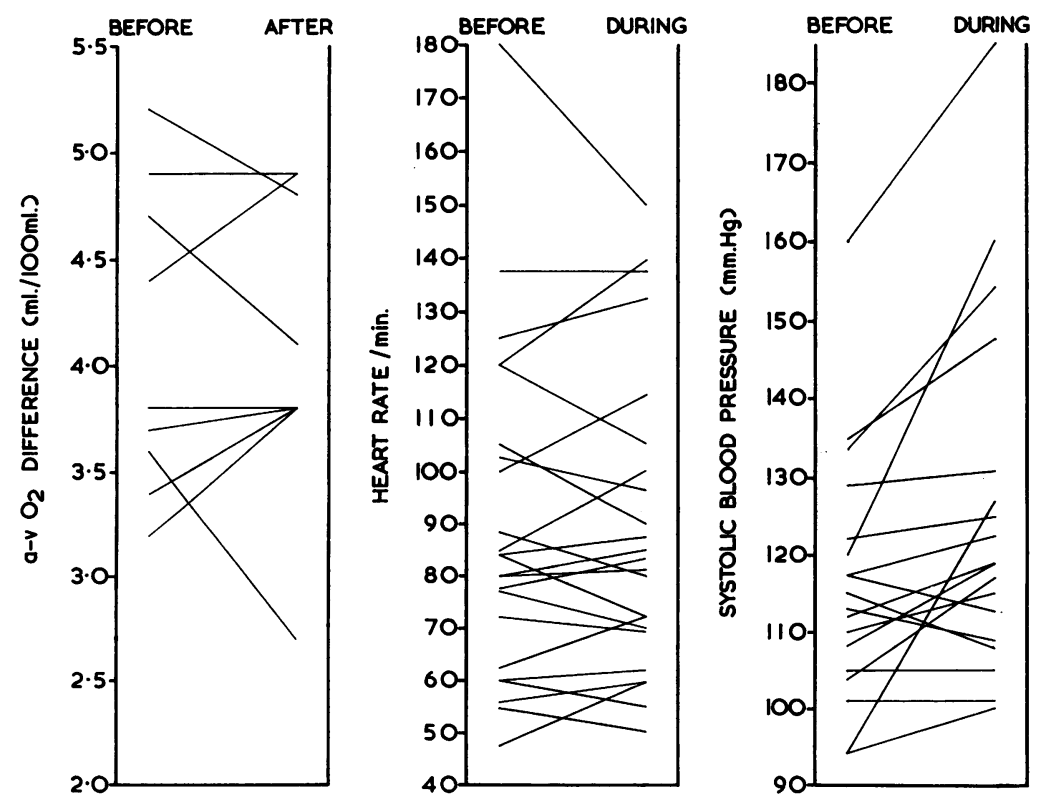

FIG. 7. -Changes in arteriovenous oxygen difference, heart rate, and systolic blood pressure occurring in association with suprasternal puncture. 
TABLE

Mortality in Suprasternal Puncture

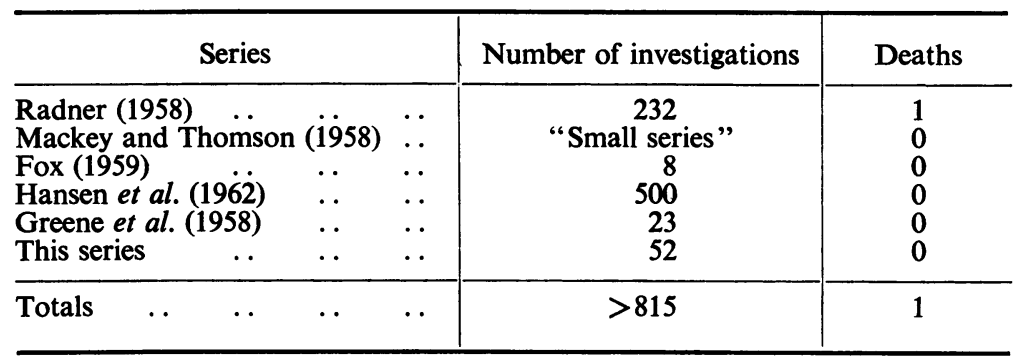

In addition, the needle used should be large enough to permit the passage of a catheter into the left ventricle and the injection of contrast medium for selective angiocardiography. It can be stated immediately that suprasternal puncture does not fulfil the last criterion and attempts to use a larger needle have been unsuccessful (Fox, 1959).

Of the other criteria mentioned, low mortality and morbidity rates are of paramount importance, and in this respect suprasternal puncture compares favourably with other procedures. In five series, listed in the Table, there has been 1 death in over 800 investigations. This was a patient who developed hæmopericardium after repeated administration of beparin; this complication was not diagnosed and the patient died on the third day after investigation. This can be compared with the reports, reviewed by Crymes et al. (1959), of 15 deaths in 2178 transthoracic left atrial punctures and 2 deaths in 1472 left atrial punctures by the bronchoscopic technique. The over-all experience with transseptal left atrial puncture is smaller; Brockenbrough, Braunwald, and Ross (1962) have published a series of 450 cases with no mortality but as further experience accumulates it seems that this procedure is by no means without risk (Adrouny et al., 1963). The incidence of complications is more difficult to determine but in this series only one of the seven patients with complications gave any cause for anxiety and in the series of Hansen et al. (1962), although 48 patients had complications, in 4 only were they significant; these were pneumothorax with loss of consciousness, pulmonary infarction, transitory paresis of the left hand, and pulmonary œdema. In the other series quoted in the Table, Radner (1958) reported no complications and Mackey and Thomson (1958) mention "few complications and those only minor ones". In contrast, Fox (1959) emphasized the dangers of the procedure and quoted a patient who developed a large mediastinal hæmatoma. However, he had been using a much larger needle than that recommended by Radner and there seems little doubt that the thinness of the needle is the main reason for the safety of suprasternal puncture. Hamer and Dow (1961) suggest that the use of such a long thin needle makes it difficult to obtain undamped records but this has not been our experience.

Although the needle entered the aorta, pulmonary artery, and left atrium in rather less than half the patients in this series, the fact that a catheter had already been introduced into the pulmonary artery obviated the need for prolonged attempts to puncture that vessel; in only one patient was the main purpose of the investigation not achieved. The actual passage of the needle rarely occupied more than five minutes, and, in fact, the ease and speed with which the procedure can be performed are such that on two occasions it was carried out on patients who were considered to be unfit for right heart catheterization on account of severe heart failure. There seems little doubt that, on this score, the suprasternal approach is superior to other methods of left atrial puncture.

With regard to the disturbing effect of the procedure on the stable state, there is very little information available on this aspect of other methods of left atrial puncture. Direct comparison of these methods with suprasternal puncture is therefore not possible. However, there was no consistent change in arteriovenous oxygen difference after suprasternal puncture (Fig. 7), and this may be compared with the finding of a widening of the arteriovenous oxygen difference in 9 out of 13 
patients investigated by left ventricular puncture (P. R. Fleming, 1963, unpublished observations). - The use of this parametei as an index of cardiac output is not entirely justifiable but it is probable

that it does reflect gross changes in blood flow. The pulse rate in most cases was little changed by suprasternal puncture and much the same was found in the patients having left ventricular puncture. The systolic blood pressure, on the other hand, was fairly consistently, but not usually markedly, raised during suprasternal puncture; similar and rather more marked changes occurred during left ventricular puncture. As Hamer and Dow (1961) pointed out, changes in cardiac output and heart rate do not affect the calculated valve area, provided that all the relevant information is obtained simultaneously; changes in blood pressure, however, are likely to alter the degree of mitral regurgitation (Braunwald, Welch, and Morrow, 1958) and, in this respect, suprasternal puncture is less than ideal. Although no data are available, it is probable that the same criticism applies to the transthoracic and bronchoscopic approaches; the effect of transseptal catheterization is less predictable.

\section{SUMMARY}

Suprasternal puncture has been carried out in 52 patients in the past four years. The technique is described in detail and the instrumentation which has been developed for combined left and right heart catheterization is also described.

The complications and the deviations from the resting state occurring in association with the procedure are discussed. It is concluded that suprasternal puncture is a simple and safe method of recording left atrial and other intravascular pressures.

We wish to thank Dr. Percy Cliffe for his invaluable assistance in the investigation of these patients and for his help in the preparation of this paper. We also thank Dr. C. J. Gavey for his encouragement during the development of this technique. Our thanks are also due to him and to several other colleagues for referring patients for investigation and to the staff of the Department of Medical Photography and Illustration, of Westminster Medical School, for the illustrations.

\section{REFERENCES}

Adrouny, Z. A., Sutherland, D. W., Griswold, H. E., and Ritzmann, L. W. (1963). Complications with transseptal left heart catheterization. Amer. Heart J., 65, 327.

Braunwald, E., Welch, G. H., and Morrow, A. G. (1958). The effects of acutely increased systemic resistance on the left atrial pressure pulse: a method for the clinical detection of mitral insufficiency. J. clin. Invest., $37,35$.

Brock, R., Milstein, B. B., and Ross, D. N. (1956). Percutaneous left ventricular puncture in the assessment of aortic stenosis. Thorax, 11, 163.

Brockenbrough, E. C., Braunwald, E., and Ross, J. (1962). Transseptal left heart catheterization. A review of 450 studies and description of an improved technic. Circulation, 25, 15.

Crymes, T. P., Fish, R. G., Smith, D. E., and Takaro, T. (1959). Complications of transbronchial left atrial puncture. Amer. Heart J., 58, 46.

Fox, S. M. (1959). Pretracheal left heart catheterization: difficult technic with some advantages. Circulation, 20, 696 .

Greene, D. G., Sharp, J. T., Griffith, G. T., Bunnell, I. L., and Macmanus, J. E. (1958). Surgical applications of anterior percutaneous left heart puncture. Surgery, $\mathbf{4 3}, 1$.

Hamer, N. A. J., and Dow, J. W. (1961). Selection of techniques for the measurement of left heart pressures. Brit. Heart J., 23, 317.

Hansen, A. T., Fabricius, J., Pedersen, A., and Sandøe, E. (1962). Suprasternal puncture of the left atrium and the great vessels. Experience from 500 punctures. Amer. Heart J., 63, 443.

- Hansen, P. F., Sandøe, E., and Winkler, K. (1961). Percutaneous diagnostic punctures of the heart and great vessels. Acta med. scand., 169, 273.

Keele, C. A., and Neil, E. (1961). Samson Wright's Applied Physiology, 10th ed., pp. 141 and 144. Oxford University Press, London.

Mackey, W. A., and Thomson, R. M. (1958). Combined Radner and Brock techniques for recording left heart pressures. In Proceedings of the British Cardiac Society. Brit. Heart J., 20, 585.

Odman, P. (1959). The radiopaque polythene catheter. Acta radiol. (Stockh.), 52, 52.

Radner, S. (1953). Method for recording aortic pressure pulses. Scand. J. clin. Lab. Invest., 5, 129.

- (1954a). Suprasternal puncture of the left atrium for flow studies. Acta med. scand., 148, 57. (1954b). Suprasternal puncture of the pulmonary artery. Acta med. scand., 148, 161 .

(1954c). Suprasternal puncture technique for left heart flow studies. Acta chir. scand., 108, 54.

(1955). Extended suprasternal puncture technique. Acta med. scand., 151, 223.

- (1958). Extended suprasternal puncture technique for flow studies in 135 cases of mitral valvular disease. In Circulation: Proc. Harvey Tercentenary Congress, London, 1957, ed. J. McMichael, p. 250. Blackwell, Oxford.

Seldinger, S. I. (1953). Catheter replacement of the needle in percutaneous arteriography; a new technique. Acta radiol. (Stockh.), 39, 368. 